\title{
Commande adaptative multivariable numérique et sa réalisation par microprocesseur Application à la régulation de vitesse d'un groupe hydroélectrique
}

\author{
Multivariable adaptative numerical control \\ and how to achieve it with a microprocessor \\ Application to speed regulation \\ of a hydroelectric generating set
}

\author{
Ye Luqing et Serge Maurin
}

Departement EDF - Direction des Etudes et Recherches

\section{Introduction}

Depuis ces dernières années la théorie de la commande adaptative se développe rapidement. Nos efforts sont consacrés plutôt à la liaison entre la théorie et la pratique.

En ce qui concerne la régulation et la commande du groupe hydro-électrique, jusqu'à présent c'est toujours le régulateur classique PID qui est utilisé. Ses inconvénients sont évidents :

1) Chaque centrale hydro-électrique a ses particularités en ce qui concerne la chute, le débit, la longueur de la conduite forcée, le type de turbine et de générateur, les caractéristiques du réseau électrique si c'est un réseau isolé, etc. Pour le régulateur PID, il est très difficile de tenir compte des particularités du système à commander.

2) Les caractéristiques de turbines hydrauliques étant non-linéaires (Fig. 1), il est possible d'établir des équations linéaires, mais les matrices de coefficients sont différentes pour chaque point de fonctionnement [4].

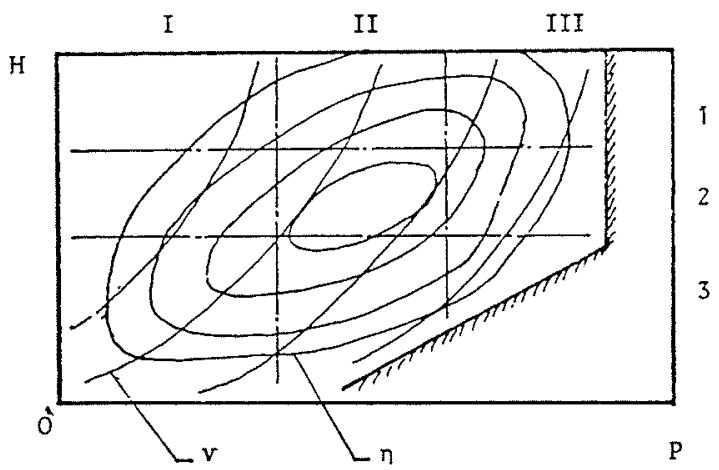

Figure 1 - Non-linéarité des caractéristique des turbines hydrauliques.
Pour le régulateur PID, la non-linéarité n'est pas prise en compte. Une fois les paramètres du régulateur adaptés à un point de fonctionnement particulier, ils sont utilisés dans tout le champ de fonctionnement. Evidemment, ce n'est pas rationnel. D'ailleurs, les caractéristiques subiront peut-être d'autres perturbations.

L'idée fondamentale de la commande adaptative est que la commande (ou les paramètres du régulateur) soit adaptée aux changements des caractéristiques du système à commander et à la perturbation éventuelle pour que les performances soient toujours optimales. On va appliquer cette méthode à la commande et à la régulation de vitesse du groupe hydro-électrique.

Par ailleurs, on voudrait utiliser le microprocesseur pour réaliser la commande adaptative.

Il faut donc prendre un compromis entre la performance et la complexité. D'une part, et fondamentalement, la performance devrait être améliorée par rapport à la méthode classique et existante, d'autre part la structure de commande proposée devrait être simple et réalisable avec le matériel actuel -- le microprocesseur.

1. Schéma de la commande adaptative avec modèles de référence

Irving E. et Dang Van Mien [3] ont résumé l'idée directrice de la commande adaptative avec modele de référence en trois points :

1) la propriété de commandabilité paramétrique des régulateurs ;

2) l'idée de modèles de référence ;

3) le principe de suffisance du comportement externe.

Ce qui veut dire que l'on propose un modèle de référence dont la sortie est bien la sortie désirée du procédé. Lorsque le procédé subit des perturbations extérieures ou paramétriques, le système de commande modifie 
adaptativement les paramètres du régulateur pour que la sortie du procédé s'approche le plus vite possible de la sortie désirée en suivant un comportement dynamique proposé.

La figure 2 montre le schéma de la commande adaptative à modèle de référence paralléle-série .

Le modèle de référence parallèle est en effet celui d'asservissement qui fixe le comportement de la sortie par rapport au changement de consigne, et le modèle de référence série est celui de régulation. Souvent, la sortie peut subir de grandes perturbations, par exemple pour le groupe hydro-électrique, quand il y a un grand changement de la charge, la fréquence varie considérablement. Dans ce cas-là, si on voulait éliminer instantanément l'écart entre le procédé et le modèle de référence parallèle $\epsilon_{r}$, la commande bougerait brusquement. Cela apporterait même de l'instabilité. Dans ce sens-là, le modèle de référence série est en réalité un filtre.

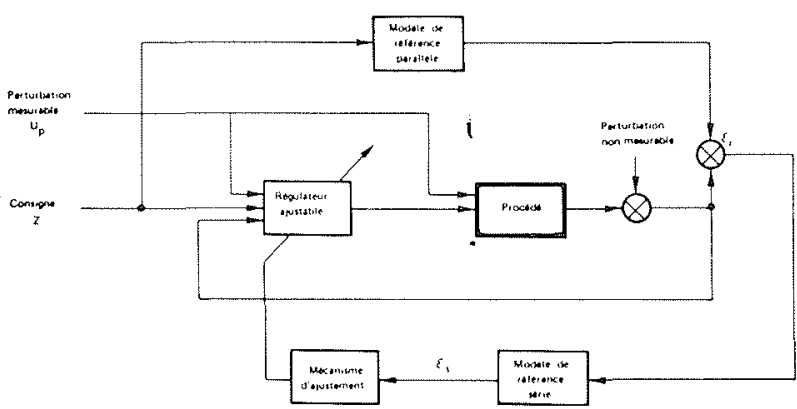

Figure 2 - Système adaptatif avec modèle de référence parallèlesérie en tenant compte de la perturbation mesurable.

Pour le problème de la commande du groupe hydroélectrique, le motif principal du changement de vitesse (sortie) est la variation de charge. Depuis ces dernières années, on a fait beaucoup de recherches sur la prévision de charge. On sait que la prévision de charge de courte durée atteint une précision suffisante. Donc, dans le système proposé (Fig. 2), on prend en compte la variation de charge prévue comme perturbation mesurable. Cela veut dire que la commande comprend la composante anticipation. Bien sûr il existe encore la pertur. bation non mesurable (y compris l'écart de prévision, la perturbation paramétrique, etc.). On va diminuer et même annuler son influence grâce au modèle de référence et au mécanisme d'ajustement.

\section{Modèle simplifié du groupe hydro-électrique hydro-électrique}

Le groupe hydro-électrique est schématisé par la figure 3. Supposons que le groupe fonctionne dans un réseau isolé, et se charge principalement de la régulation de fréquence du réseau. Notre objectif est que la fréquence du réseau soit la plus proche possible de la consigne quand le réseau subit des perturbations, surtout celle qui résulte de la variation brutale de charge.

Ainsi, c'est la fréquence qui est la valeur réglée et qui constitue la sortie du système. Pour généraliser le pro- blème, nous considérons que la turbine (par exemple Kaplan) a deux organes de réglage : vannages et pales. Ils sont commandés par leur distributeur respectif. Le servomoteur et son distributeur composent un système de $1^{\text {er }}$ ordre (cf. [4]). Le distributeur est commandé par l'actionneur qui peut être considéré comme un amplificateur linéaire à cause de la constante de temps, petite par rapport aux autres constantes. Donc, on prend pour commander $U$, l'ouverture du distributeur du servomoteur des vannages et celle des pales.

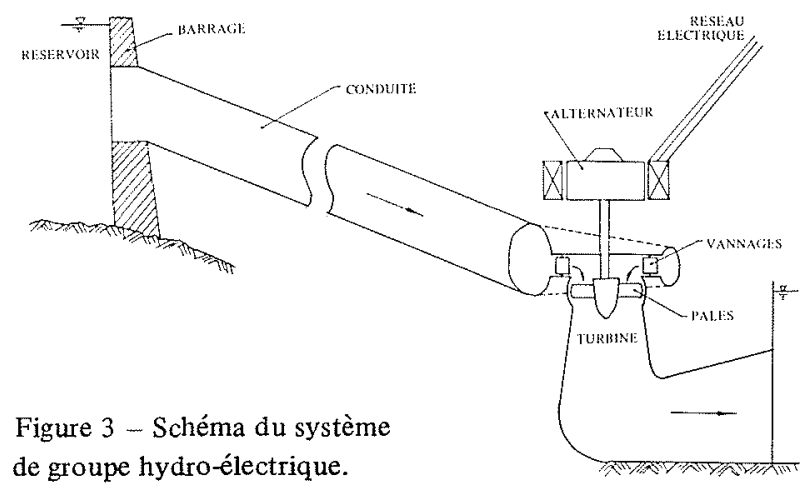

Si l'on voulait obtenir non seulement l'optimalité dynamique, mais encore l'optimalité statique ou énergétique, on devrait réaliser à l'état statique la conjugaison entre l'ouverture des vannages et celle des pales qui correspond aux meilleurs rendements.

Pour cela, on ajoute la deuxième sortie :

$$
Y_{2}=S_{1}-\gamma S_{2}
$$

Ici, $S_{1}, S_{2}$ sont respectivement l'ouverture des vannages et celle des pales, $\gamma$ est le coefficient de conjugaison entre les deux. Certes, $\gamma$ varie avec les points de fonctionnement. On peut composer pour $\gamma$ une table de correspondance dans la mémoire avec les paramètres statiques. La consigne de $Y_{2}$ est généralement nulle.

Ainsi, la sortie $Y$ et l'entrée $U$ sont toutes les deux d'ordre 2. Cela constitue un système multivariable.

D'une façon générale, le système est régi par l'équation suivante en cas discret :

$$
\begin{array}{r}
Y(k)=\sum_{i=1}^{n} A_{i} Y(k-i)+\sum_{j=1}^{m} B_{j} U(k-j)+ \\
+\sum_{p=1}^{l} F_{p} U_{p}(k-p)
\end{array}
$$

avec

$k$ nombre de périodes d'échantillonnage, c'est-à-dire le temps ;

$A_{i}(i=1 \sim n), B_{j}(j=1 \sim m)$ - matrices de coefficients d'ordre $2 \times 2$;

$F_{p}(p=1 \sim l)$ - vecteurs de coefficients d'ordre 2 ;

$U_{p}$ - perturbation mesurable (prévision de variation de charge). 
D'une façon générale $A_{i}, B_{j}, F_{p}$ peuvent être déterminés par identification. En théorie, semble-t.il, si $n$, $m, l$ étaient plus élevés, le modèle serait plus proche du procédé. Par conséquent, le temps de calcul en temps réel augmente. On doit donc économiser le mieux possible le temps de calcul tout en assurant les performances exigées. Dans la commande adaptative avec modèle de référence, la comparaison du comportement externe est traitée. Ainsi, on a la possibilité de simplifier le modèle du procédé à condition que la sortie du procédé soit la plus proche possible de celle du modèle de référence.

Supposons que le modèle soit du second ordre :

$Y(k)=A_{1}(k-1) Y(k-1)+A_{2}(k-1) Y(k-2)+$

$+B_{1}(k-1) U(k-1)+F_{1}(k-1) U_{p}(k-1)$

(2.3) est la simplification de (2.2). La simulation montre qu'une telle simplification est admissible [cf. VII].

\section{Structure du modèle de référence parallèle- série}

La figure 2 montre le système adaptatif avec modèle de référence parallèle-série en tenant compte de la perturbation mesurable. Cela fait, on aborde maintenant la structure concrète du modèle parallèle-série.

Posons comme hypothèse que la commande prenne la forme suivante :

$$
\begin{aligned}
\lambda_{u}(k) U(k)=- & \lambda_{1}(k) Y(k)-\lambda_{2}(k) Y(k-1)+ \\
& +\lambda_{p}(k) U_{p}(k)+\lambda_{Z}(k) Z(k)
\end{aligned}
$$

qui montre que la commande $U(k)$ dépend non seulement du comportement de $Y(k), Y(k-1)$, de la consigne $Z(k)$, mais encore de la perturbation mesurable $U_{p}$ qui constitue le feed forward.

En substituant (3.1) à (2.3), on a :

$$
\begin{aligned}
Y(k) & =\left[A_{1}(k-1)-B_{1}(k-1) \lambda_{u}^{-1}(k-1) \lambda_{1}(k-1)\right] Y(k-1)+ \\
& +\left[A_{2}(k-1)-B_{1}(k-1) \lambda_{u}^{-1}(k-1) \lambda_{2}(k-1] Y(k-2)+\right. \\
& +\left[F_{1}(k-1)+B_{1}(k-1) \lambda_{u}^{-1}(k-1) \lambda_{p}(k-1)\right] U_{p}(k-1)+ \\
& +B_{1}(k-1) \lambda_{u}^{-1}(k-1) \lambda_{Z}(k-1) Z(k \quad 1)
\end{aligned}
$$

Supposons que le modèle de référence parallèle s'exprime comme suit :

$Y_{r}(k)=A_{1 r} Y_{r}(k-1)+A_{2 r} Y_{r}(k-2)+\beta_{r} Z(k-1)$

montrant la sortie désirée. Bien sûr, elle ne doit jamais dépendre de la perturbation.

Si l'on a, à tout moment, les relations suivantes :

$$
\begin{aligned}
& \lambda_{u}(k-1)=B_{1}(k-1) \\
& \lambda_{Z}(k-1)=\beta_{r} \\
& \lambda_{1}(k-1)=A_{1}(k-1)-A_{1 r} \\
& \lambda_{2}(k-1)=A_{2}(k-1)-A_{2 r} \\
& \lambda_{p}(k-1)=-F_{1}(k-1)
\end{aligned}
$$

la sortie du procédé $Y(k)$ est toujours identique à celle du modèle de référence parallèle $Y_{r}(k)$, c'est-à-dire :

$$
Y(k) \equiv Y_{r}(k)
$$

Bien entendu, ce n'est qu'un cas idéal. En réalité, l'écart existe toujours entre les deux :

$$
\epsilon_{r}(k)=Y_{r}(k)-Y(k)
$$

En retranchant (2.3) de (3.3) et en considérant (3.5), on obtient :

$$
\begin{aligned}
& \epsilon_{r}(k)-A_{1 r} \epsilon_{r}(k-1)-A_{2 r} \epsilon_{r}(k-2) \\
&=\left[A_{1 r}-A_{1}(k-1)\right] Y(k-1)+ \\
& \quad+\left[A_{2 r}-A_{2}(k-1)\right] Y(k-2)- \\
&-B_{1}(k-1) \cdot U(k-1)-F_{1}(k-1) U_{p}(k-1)+ \\
& \quad+\beta_{r} Z(k-1)(3.6)
\end{aligned}
$$

Si l'on choisit le modèle de référence série comme suit :

$$
\epsilon_{s}(k)=\epsilon_{r}(k)-A_{1 r} \epsilon_{r}(k-1)-A_{2 r} \epsilon_{r}(k-2)
$$

on a enfin :

$$
\begin{aligned}
\epsilon_{s}(k)= & {\left[A_{1}-A_{1}(k-1)\right] Y(k-1)+} \\
+\left[A_{2 r}\right. & \left.-A_{2}(k-1)\right] Y(k-2)-B_{1}(k-1) U(k-1) \\
& -F_{1}(k-1) U_{p}(k-1)+\beta_{r} Z(k-1)
\end{aligned}
$$

Ainsi (3.3), (3.7) et (3.8) sont respectivement les modèles parallèle et série.

\section{Ajustement optimal en utilisant le filtre de Kalman et l'idée de "Self Tuning Regulator"}

Notre discussion se divise en deux étapes. Dans la première étape, la problème en question est considéré comme celui de l'estimation des parametres. On utilise le principe du filtre de Kalman pour résoudre ce problemme. Dans la deuxième étape, sur la base de la discussion de la première étape et en utilisant l'idée de "self tunign regulator" d'Aströme [2], on recherche la commande optimale.

On commence par la première étape.

Notre objectif est d'annuler l'écart $\epsilon_{s}(k)$ à la sortie du modele de référence série tout en ajustant les paramètres du régulateur. D'abord, on transforme ce problème en celui de l'estimation de paramètres.

On exprime les paramètres à estimer comme suit :

$$
\begin{aligned}
& P(k)= \\
& {\left[A_{1 r}-A_{1}(k), A_{2 r}-A_{2}(k),-B_{1}(k),-F_{1}(k)\right]}
\end{aligned}
$$

Dans notre problème, $P(k)$ est une matrice d'ordre $2 \times 7$.

Dans l'environnement stochastique, on a :

$$
P(k)=P(k-1)+\pi
$$


$\pi$ étant une perturbation aléatoire dont les éléments sont considérés comme bruit blanc.

Dans ce cas-là, (3.8) peut s'exprimer comme suit :

$$
\epsilon_{s}(k)=P(k-1) X(k-1)+\beta_{r} Z(k-1)+e(4.3)
$$

avec :

$$
X^{T}(k)=\left[Y^{T}(k), Y^{T}(k-1), U^{T}(k), U_{p}(k)\right](4.4)
$$

$X(k)$ étant un vecteur d'ordre 7 .

L'indice supérieur $T$ est le signe de transposition de matrice, et " $e$ " est une perturbation aléatoire dont les éléments sont considérés comme bruit blanc. Pour bien comparer avec la forme classique du filtre de Kalman, on met (4.2) et (4.3) sous la forme suivante :

$$
\begin{gathered}
P^{T}(k)=P^{T}(k-1)+\pi^{T} \\
\epsilon_{s}^{T}(k)=X^{T}(k-1) P^{T}(k-1)+Z^{T}(k-1) \beta_{r}^{T}+e^{T}
\end{gathered}
$$

Le filtre de Kalman donnant l'estimation optimale sur la base du minimum de la covariance de l'écart, on obtient les résultats suivants :

$$
\begin{aligned}
& \hat{P}^{T}(k)=\hat{P}^{T}(k-1)+K^{T}(k-1)\left[\hat{\epsilon}_{s}^{T}(k)-\epsilon_{s}^{T}(k)\right] \\
& \hat{\epsilon}_{s}^{T}(k)=X^{T}(k-1) \cdot \hat{P}^{T}(k-1)+Z^{T}(k-1) \cdot \beta_{r}^{T}
\end{aligned}
$$

avec :

$$
\begin{gathered}
K^{T}(k)=V(k) \cdot X(k) \cdot\left[R+X^{T}(k) V(k) X(k)\right]^{-1} \\
V(k+1)=V(k)+Q \\
-V(k) X(k)\left[R+X^{T}(k) V(k) X(k)\right]^{-1} X^{T}(k) V(k) \\
R=E\left[e^{T} e\right] \\
Q=E\left[\pi^{T} \pi\right]
\end{gathered}
$$

$E$ étant l'espérance mathématique.

Supposons que les éléments de " $e$ " et " $\pi$ " soient indépendants entre eux et par rapport au temps. Il est possible ainsi de diviser le problème (4.5), (4.6) en deux parties :

$$
\begin{aligned}
& p_{i}^{T}(k)=p_{i}^{T}(k-1)+\pi_{i}^{T} \quad(i=1,2) \\
\epsilon_{s i}(k)= & X^{T}(k-1) p_{i}^{T}(k-1)+ \\
& +Z^{T}(k-1) \cdot \beta_{i}^{T}+e_{i} \quad(i=1,2)
\end{aligned}
$$

Dans ce cas-là, pour $i=1,2$, le filtre de Kalman prend les formes suivantes:

$$
\begin{gathered}
\hat{p}_{i}^{T}(k)=\hat{p}_{i}^{T}(k-1)+K_{i}^{T}(k-1)\left[\epsilon_{s_{i}}^{T}(k)-\dot{\epsilon}_{s_{i}}^{T}(k)\right] \\
\hat{\epsilon}_{s_{i}}(k)=X^{T}(k-15) \cdot \hat{p}_{i}(k-1)+Z^{T}(k-1) \cdot \beta_{r_{i}}^{T}
\end{gathered}
$$

avec:

$$
K_{i}^{T}(k)=V_{i}(k) \cdot X(k) \cdot\left[R_{i}+X^{T}(k) V_{i}(k) X(k)\right]^{-1}
$$

$$
\begin{aligned}
& V_{i}(k+1)=V_{i}(k)+Q_{i} \\
& -V_{i}(k) X(k)\left[R_{i}+X^{T}(k) V_{i}(k) X(k)\right]^{-1} X^{T}(k) V_{i}(k)
\end{aligned}
$$

$$
\begin{aligned}
R_{i} & =E\left[e_{i}^{2}\right] \\
Q_{i} & =E\left[\pi_{i}^{T} \pi\right]
\end{aligned}
$$

En raison de l'indépendance des éléments de " $e$ " et " $\pi$ ", $Q_{i}$ est une matrice diagonale d'ordre $7 \times 7 . R_{i}$ est un scalaire.

Le signe “„” pour $\hat{\rho}_{i}$ et $\hat{\epsilon}_{i}$ signifie que les paramètres et l'écart estimés sont optimaux.

L'estimation optimale des paramètres est ainsi effectuée. On entre donc dans la deuxième étape, celle de la détermination de la commande optimale.

L'idée fondamentale de "self tuning regulator" est de chercher une commande pour que l'écart estimé soit identique à zéro, de sorte que :

$$
\hat{\epsilon}_{s}(k) \equiv 0
$$

En faisant l'hypothèse que (4.4) est vérifiée, (3.1) peut s'écrire :

$$
\lambda(k-1) X(k-1)+\lambda_{Z}(k-1) Z(k-1)=0
$$

avec :

$$
\lambda(k)=\left[-\lambda_{1}(k),-\lambda_{2}(k),-\lambda_{u}(k), \lambda_{p}(k)\right]
$$

En comparant (4.8) et (4.22), si

et

$$
\begin{gathered}
\lambda(k-1)=\hat{p}(k-1) \\
\lambda_{Z}(k-1)=\beta_{r} \\
\hat{\epsilon}_{s}(k) \equiv 0 \quad \text { est satisfait } .
\end{gathered}
$$

On sait que :

$$
\epsilon_{s}(k)=\hat{\epsilon}_{s}(k)+\delta(k)
$$

si $\delta(k)$ est l'écart de prédiction.

Lorsque $\hat{\epsilon}_{s}(k)$ est nul, $\epsilon_{s}(k)$ va prendre pour valeur l'écart de prédiction $\delta(k)$. Ce qui veut dire que $\epsilon_{s}(k)$ ainsi obtenu est minimal.

D'après tout ce qui précède, on a les relations suivantes :

$$
\left.\begin{array}{c}
V_{i}(k+1)=V_{i}(k)+Q_{i}-V_{i}(k) X(k) \\
{\left[R_{i}+X^{T}(k) V_{i}(k) X(k)\right]^{-1} X^{T}(k) V_{i}(k)} \\
K_{i}^{T}(k)=V_{i}(k) X(k)\left[R_{i}+X^{T}(k) V_{i}(k) X(k)\right]^{-1} \\
\lambda_{i}^{T}(k)=\lambda^{T}(k-1)+K_{i}^{T}(k-1) \epsilon_{s_{i}}(k) \quad(i=1,2)
\end{array}\right\}
$$

Une fois $\lambda^{T}(k)$ obtenu, la commande optimale $U(k)$ peut être calculée par (4.22) et (4.23).

\section{Contraintes}

Dans notre problème, il existe quelques contraintes : 


\section{1) Contraintes sur l'ouverture des vannages et celle des pales}

D'une part, l'ouverture a des valeurs maximales et minimales; d'autre part quand la turbine fonctionne au-dessous de la chute nominale, la contrainte est déterminée par la puissance maximale possible afin d'avoir un rendement raisonnable. Donc :

$$
S_{i_{\min }} \leqslant S_{i} \leqslant S_{i_{\max }} \quad(i=1,2)
$$

$S_{i}(i=1,2)$ représente l'ouverture des vannages $(i=1)$ et celle des pales $(i=2)$. $S_{i \max }$ et $S_{i_{\min }}$ sont des valeurs limites, et peuvent être variables avec la chute.

\section{2) Contraintes sur la pression hydraulique}

C'est extrêmement important pour la sécurité de la centrale hydraulique. Essentiellement la pression est proportionnelle à la vitesse de fermeture et d'ouverture des vannages et des pales.

Ainsi, on a :

$$
\dot{S}_{i_{\max }} \geqslant \dot{S}_{i} \geqslant \dot{S}_{i_{\min }} \quad(i=1,2)
$$

$\dot{S}_{i_{\max }}$ et $\dot{S}_{i_{\min }}$ sont les limites de vitesse d'ouverture et de fermeture, de signe différent.

Comme il a été vu au [4], le servomoteur hydraulique et son distributeur liés par une réaction locale composent un système du premier ordre. Pour simplifier le calcul en temps réel et à cause des petites constantes de temps du système en question, on peut approximative. ment limiter $S_{i}$ et $\dot{S}_{i}(i=1,2)$ respectivement $\operatorname{par} U_{i}$ et $\dot{U}_{i}(i=1,2)$, donc :

$$
\begin{array}{ll}
U_{i_{\max }} \geqslant U_{i} \geqslant U_{i_{\min }} & (i=1,2) \\
\dot{U}_{i_{\max }} \geqslant \dot{U}_{i} \geqslant \dot{U}_{i_{\min }} & (i=1,2)
\end{array}
$$

$U_{i_{\max }}$ et $U_{i_{\min }}$ ainsi que $\dot{U}_{i_{\max }}$ et $\dot{U}_{i_{\min }}$ sont des valeurs limitées.

\section{Algorithme en temps réel}

La séquence de calcul pour chaque période d'échantillonnage est clairement indiquée à la figure 4 . Toutes les opérations arithmétiques et logiques sont faites par microprocesseur.

La liaison entre microprocesseur et procédé est faite par interfaces, de la façon suivante :

- Le microprocesseur doit acquérir, en provenance du procédé, les valeurs suivantes : $Y(k), S_{10}(k), S_{20}(k)$, $H_{0}(k)$ qui sont mesurées. La fréquence $Y_{1}(k)$ est facilement mesurable. $S_{10}(k), S_{20}(k)$ et $H_{0}(k)$ sont des paramètres statiques à chaque instant. Ils sont nécessaires, d'une part pour consulter $\gamma$ dans le tableau prédéfini, puis pour calculer $Y_{2}(k)$ d'après $(2.1)$, d'autre part pour consulter $S_{i_{\max }}$ et $S_{i_{\min }}$ dans un autre tableau. - Le convertisseur $A / D$ transforme le signal analogique en signal numérique.

- Le microprocesseur doit recevoir à chaque instant deux ordres extérieurs : la consigne $Z$ et la prévision de la perturbation mesurable $U_{p}$.

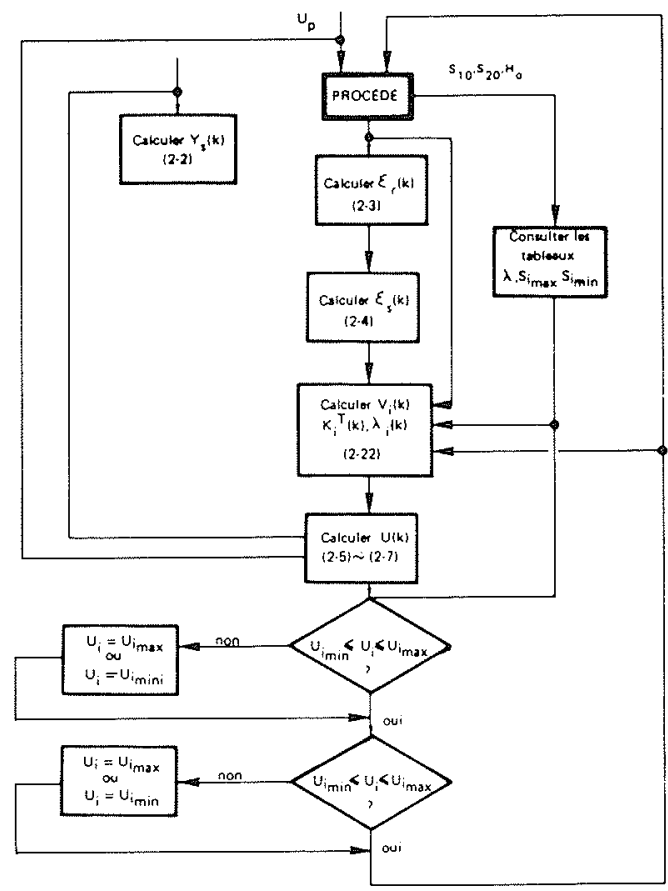

Figure 4 - Organigramme en temps réel (pour une période d'échantillonnage).

Remarque : Les chiffres dans les blocs montrent le numéro de l'expression qu'on utilise dans le pas en question.

- Par ailleurs, le microprocesseur doit transmettre au procédé la commande $U$. D'abord, le signal digital est transformé en signal analogique électrique par convertisseur $D / A$. Puis, le signal analogique électrique est transformé en signal mécanique et hydraulique par l'actionneur qui est lié directement avec le distributeur. La structure pour les vannages et pour les pales est semblable.

\section{Simulation numérique}

Tout ce qui précède et surtout l'algorithme du paragraphe précédent (Fig. 4) a permis de réaliser une simulation numérique.

Le procédé est simulé par l'équation d'état d'ordre 6 en tenant compte du coup de bélier d'onde (cf. [4]).

$$
\begin{gathered}
X(k+1)=A X(k)+B U(k)+F U_{p}(k) \\
Y(k)=C X(k)
\end{gathered}
$$

avec :

$A$ : matrice de $6 \times 6$;

$B$ : matrice de $6 \times 2$;

$F$ : vecteur de 6 ;

$C$ : matrice de $2 \times 6$.

$A, B$ et $F$ sont obtenues de la discrétisation des matrices respectives dans le cas continu (cf. [4]). L'état $X$ est un vecteur d'ordre 6 , et la sortie $Y$ est un vecteur d'ordre 2 .

L'un des deux éléments de la sortie est la vitesse ou la fréquence qui est la principale variable réglée, l'autre 
concerne la conjugaison entre vannages et pales. On a donc :

$$
C=\left[\begin{array}{rrrrrr}
1 & 0 & 0 & 0 & 0 & 0 \\
0 & 0 & 0 & 0 & 1 & -\gamma
\end{array}\right]
$$

avec $\gamma$ : coefficient de conjugaison.

Remarque : De la figure 5 à la figure 14 toutes les valeurs sont normalisées. L'abscisse est exprimée en seconde.

On va examiner et discuter les résultats obtenus.

1) Tout d'abord on va examiner les cas avec perturbation de charge en conservant la consigne nulle. On donne ici quelques cas typiques.

Les figures 5 et 6 montrent la réponse dynamique après perturbation de charge en échelon respectivement de $+10 \%$ et $-30 \%$ de la puissance nominale.

Les figures 7 et 8 indiquent des résultats dont les perturbations sont effectuées conformément au tableau I.

L'évolution de la perturbation $U_{p}$ apparaît également sur les figures 7 et 8 .

\begin{tabular}{|c|c|c|c|c|}
\hline \multicolumn{4}{|c|}{ Tableau I } \\
\hline Cas & $U_{p} \frac{d U_{p}}{d t}$ & $0 \sim 1$ & $1 \sim 3$ & $>3$ \\
\hline $\begin{array}{c}\text { (a) } \\
\text { figure } \\
7\end{array}$ & $\frac{d U_{p}}{d t}$ & 0,1 & $-0,1$ & 0 \\
\hline \multirow{2}{*}{$\begin{array}{c}\text { (b) } \\
\text { figure } \\
8\end{array}$} & $\frac{U_{p}}{d U_{p}}$ & $0,1 t$ & $0,2-0,1 t$ & $-0,1$ \\
\cline { 2 - 5 } & $\frac{d U_{p}}{d t}$ & $\sin \frac{\pi}{2} t$ & $-0,1$ \\
\hline
\end{tabular}

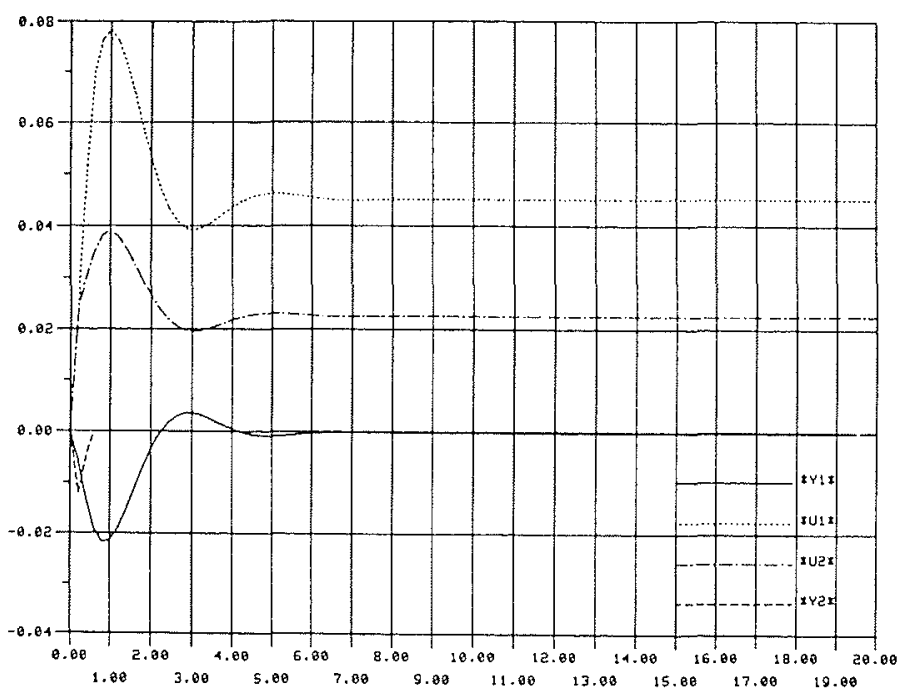

Figure 5 - Réponse dynamique dans le cas où : $U P=10 \%$ et $Z 1=0$.

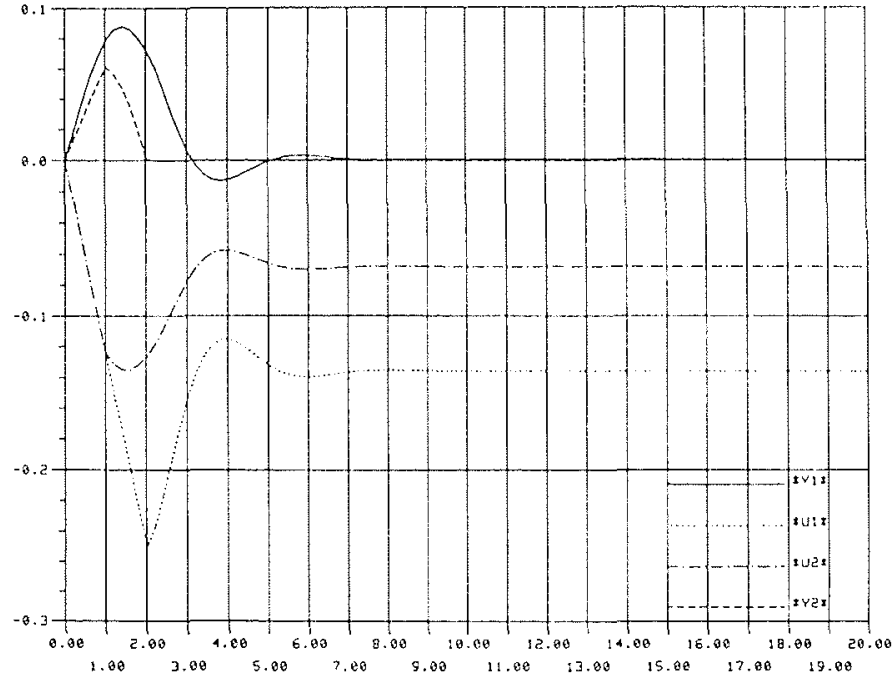

Figure 6 - Réponse dynamique dans le cas où : $U P=-30 \%$ et $Z 1=0$.

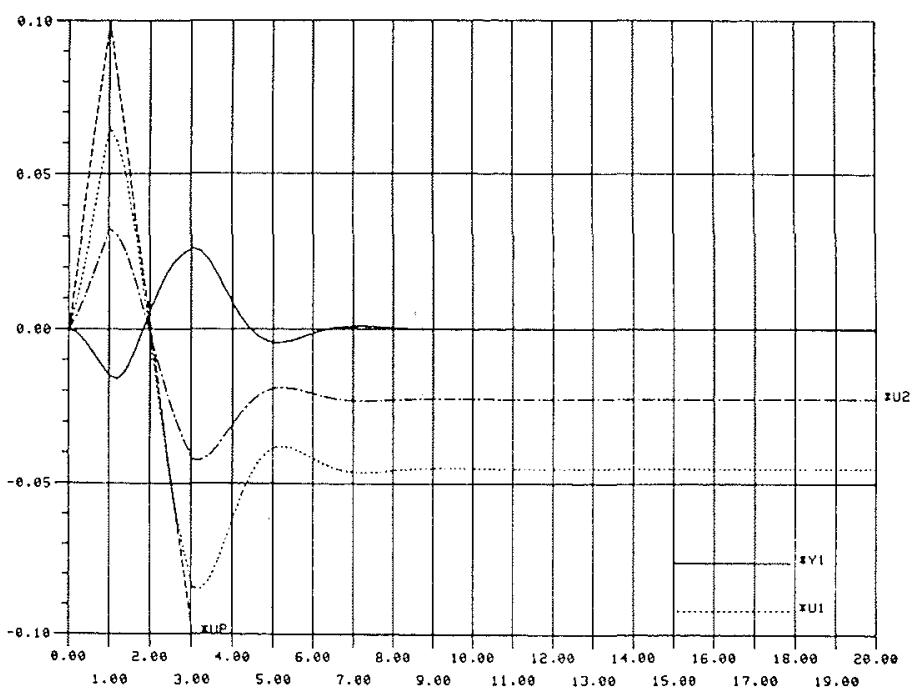

Figure 7 - Réponse dynamique dans le cas (A) de tableau I.

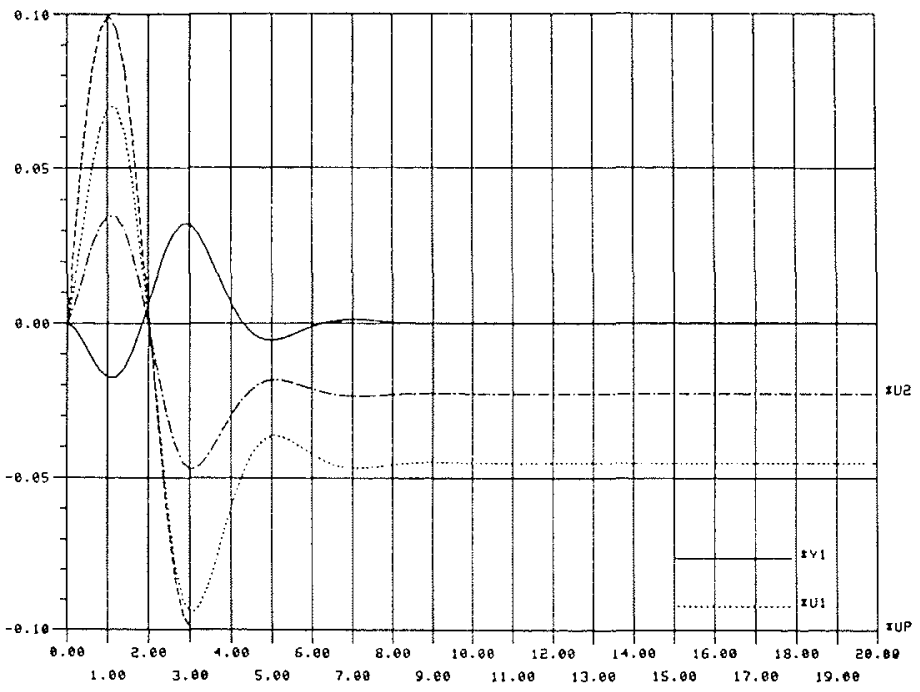

Figure 8 - Réponse lors d'une perturbation sinusoïdale : cas (B) de tableau $\mathrm{I}$. 


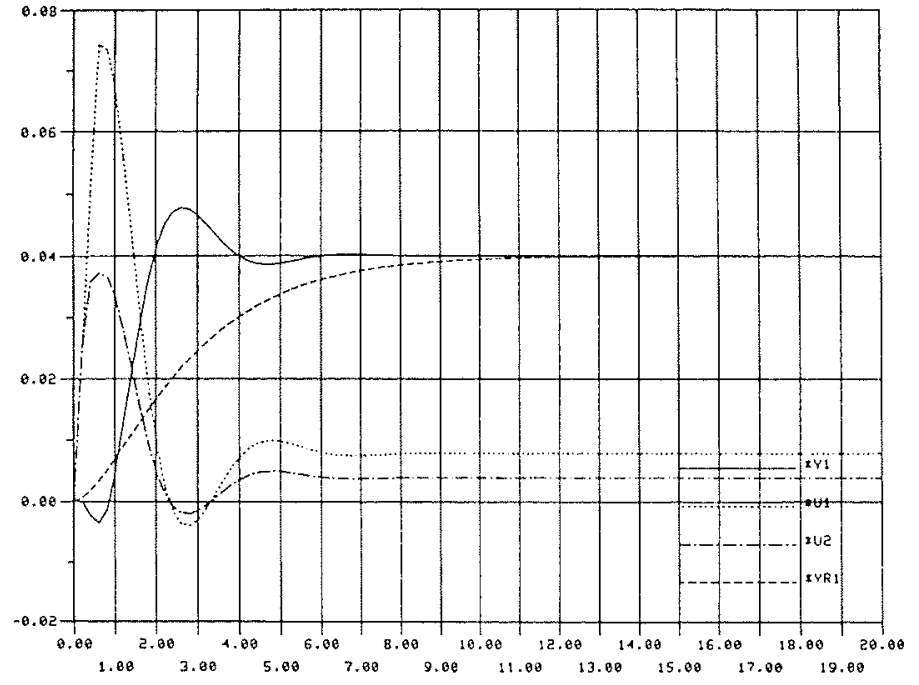

Figure 9 - Réponse dynamique dans le cas où : $U P=0$ et $Z 1=0.04$.

On s'aperçoit que pour n'importe quelle sorte de perturbation les performances sont toujours satisfaisantes.

(i) Le système est stable, avec une vitesse de convergence satisfaisante ;

(ii) La seconde sortie $Y_{2}$ évolue comme prévu. On réalise à la fois l'optimalité énergétique et l'optimalité dynamique ;

(iii) Les contraintes fonctionnent comme prévu.

2) La figure 9 montre uniquement le fonctionnement de l'asservissement, $Z_{1}=0,04(2 \mathrm{HZ})$. La sortie réelle suit bien la sortie désirée. Quant à la sortie désirée elle-même, on peut la fixer comme on le veut. Il est clair que la consigne de la seconde sortie $Z_{2}$ reste toujours nulle afin d'assurer la conjugaison entre vannages et pales.

Les figures 10 et 11 montrent les résultats lorsque le procédé subit simultanément la perturbation de charge et la modification de consigne.

La figure 10 représente le cas où : $Z_{1}=-0,04$ et $U_{p}=30 \%$, la figure 11 représente le cas où : $Z_{1}=$ $-0,04$ et $U_{p}=-30 \%$.

Donc au niveau dynamique, le système proposé assure le minimum d'écart à la sortie par rapport à la consigne, et au niveau statique, la sortie correspond toujours à la consigne. La réponse d'asservissement avec ou sans perturbation est satisfaisante dans les deux cas.

3) On va vérifier la robustesse et l'adaptabilité. On sait bien que l'identification est toujours réalisée avec une certaine approximation. Même s'il était possible d'obtenir une excellente identification, l'état du procédé pourrait changer avec le temps et avec le régime.

Pour vérifier l'adaptativité du système étudié, on a fait des essais dont on montre un exemple.

Supposons que les paramètres primitifs du procédé aient un écart, soit de $+15 \%$, soit de $-15 \%$ par rapport aux paramètres originaux. Pour avoir une idée sur les grandeurs, on montre ici des matrices discrétisées dans deux cas.

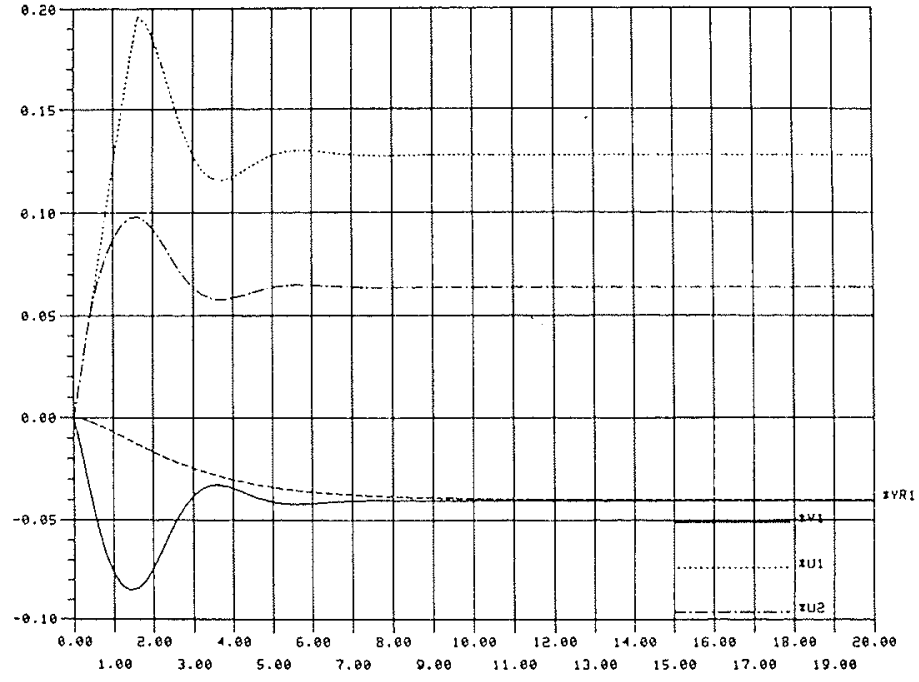

Figure 10 - Réponse dynamique dans le cas où : UP $=30 \%$ et $Z 1=-0.04$.

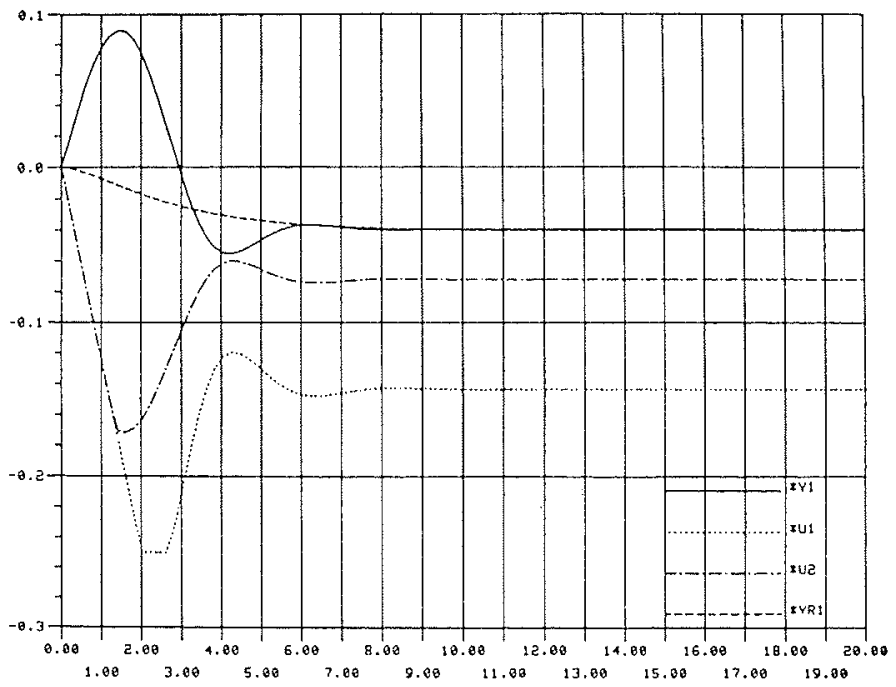

Figure 11 - Réponse dynamique dans le cas où : $U P=-30 \%$ et $Z 1=-0.04$

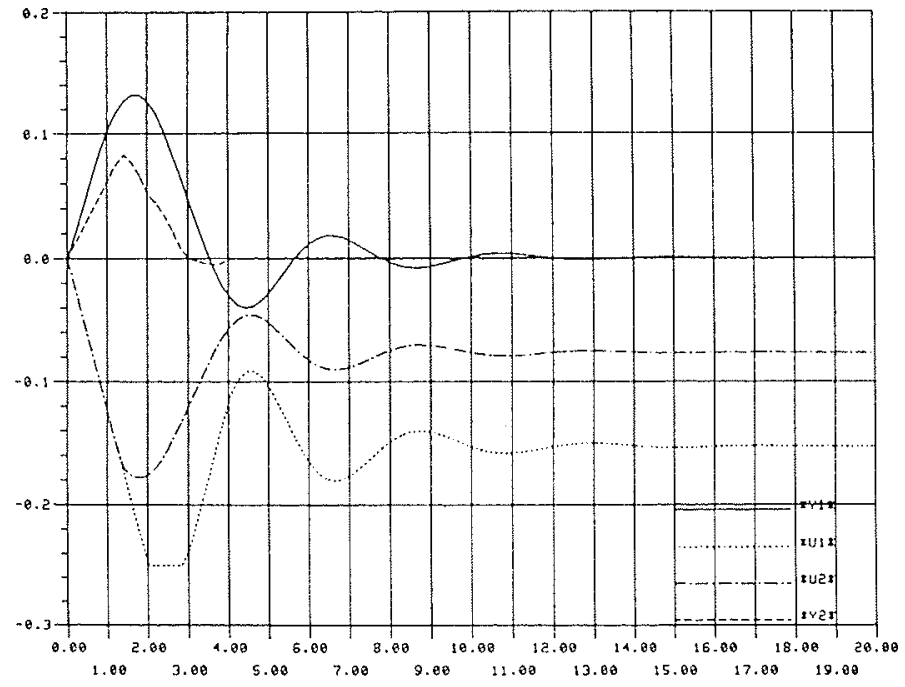

Figure 12 - Réponse du procédé modifié dans le cas où : $U P=-30 \%$ et $Z 1=0$. 
Les équations (7.4), (7.5) et (7.6) sont les matrices originales $A, B^{T}$ et $F^{T}$,

$$
\begin{aligned}
& A=\left[\begin{array}{llcccc}
0.925501 & -0.0079 & -0.003152 & 0.103033 & -0.002628 & -0.02669 \\
0.290014 & 0.346844 & 1.428496 & 0.094866 & 0.109093 & 0.248274 \\
0.0096 & -0.057973 & 0.182779 & 0.054877 & -0.020262 & -0.040918 \\
0.347769 & 0.05689 & 0.037151 & 0.277645 & 0.122808 & 0.295339 \\
0 & 0 & 0 & 0 & 0.082085 & 0 \\
0 & 0 & 0 & 0 & 0 & 0.135335
\end{array}\right] \\
& B^{T}=\left[\begin{array}{llllcc}
-0.017504 & 0.443379 & 0.054027 & 0.544688 & 2.1598 & 0 \\
-0.070331 & 0.615281 & 0.078562 & 0.736258 & 0 & 1.503765
\end{array}\right] \\
& F^{T}=\left[\begin{array}{llllll}
-0.055715 & -0.010737 & -0.001547 & -0.010689 & 0 & 0
\end{array}\right]
\end{aligned}
$$

et les équations $(7.7),(7.8)$ et $(7.9)$, les matrices modifiées $A_{ \pm 15}, B_{ \pm 15}^{T}$ et $F_{ \pm 15}^{T}$

$$
\begin{aligned}
& A_{ \pm 15}=\left[\begin{array}{cccccc}
0.887576 & -0.024659 & -0.081262 & 0.167382 & -0.017073 & -0.036162 \\
0.252378 & 0.128284 & 2.380861 & 0.456385 & 0.055667 & 0.101233 \\
0.018297 & -0.083704 & -0.192472 & 0.052706 & -0.017802 & 0.251104 \\
0.385633 & 0.106918 & 0.371969 & 0.263914 & 0.141067 & 0.25104 \\
0 & 0 & 0 & 0 & 0.082085 & 0 \\
0 & 0 & 0 & 0 & 0 & 0.135335
\end{array}\right] \\
& B_{ \pm 15}^{T}=\left[\begin{array}{cccccc}
0.063171 & 0.41416 & 0.076569 & 0.503173 & 1.83583 & 0 \\
-0.101259 & 0.588765 & 0.108648 & 0.683836 & 0 & 1.729329
\end{array}\right] \\
& F_{ \pm 15}^{T}=\left[\begin{array}{llllll}
-0.062777 & -0.014626 & -0.002513 & -0.013406 & 0 & 0
\end{array}\right]
\end{aligned}
$$

Puis on fait des essais sans modifier la structure du système de commande et ses paramètres afin de vérifier sa robustesse.

La figure 12 correspond au cas où $U_{p}=-30 \%$ et $Z_{1}=0$, la figure 13 au cas où $U_{p}=+30 \%$ et $Z_{1}=0,04$ et la figure 14 au cas où $U_{p}=-30 \%$ et $Z_{1}=-0,04$.

On peut faire des comparaisons entre le procédé original et le procédé modifié en conservant d'autres conditions inchangées pour les cas suivants :

figure 12 avec figure 6 ;
figure 13 avec figure 10;
figure 14 avec figure 11;

Après les comparaisons, on s'aperçoit que malgré d'importantes différences des paramètres du procédé, le système fonctionne bien, est stable et assure de bonnes performances. Donc, le système est robuste.

On a déjà fait une vérification statique de la robustesse. On va faire sa vérification dynamique. C'est-à-dire, lorsque les paramètres du procédé changent au cours du processus dynamique, quelle est l'adaptabilité de notre système?

On va étudier trois cas différents :

1) Le premier cas correspond au tableau II :

\begin{tabular}{|c|c|c|c|c|c|c|}
\hline \multicolumn{7}{|c|}{ Tableau II (pour les figures 15 et 16) } \\
\hline$t(\mathrm{sec})$ & $t \leqslant 1$ & $1<t \leqslant 2$ & $2<t \leqslant 3$ & $3<t \leqslant 4$ & $4<t \leqslant 5$ & $t>5$ \\
\hline $\begin{array}{c}\text { Modèle } \\
\text { du } \\
\text { procédé }\end{array}$ & $M$ & $O$ & $M$ & $O$ & $M$ & $O$ \\
\hline
\end{tabular}

La figure 15 représente la simulation pour une perturbation de la charge de $-30 \%$ en conservant la consigne constante, la figure 16 pour la perturbation de la charge $\mathrm{de}-30 \%$ et la modification de la consigne de $-2 \mathrm{~Hz}$.

2) Le deuxième cas correspond au tableau III.

En comparant les tableaux II et III, on s'aperçoit que leur configuration ainsi que la durée de chaque zone sont différentes.

La figure 17 est relative à une perturbation de la charge de $-30 \%$ sans modification de la consigne, la figure 18 à une perturbation de la charge de $-30 \%$ et une modification de la consigne de $-2 \mathrm{~Hz}$.

3) Dans le troisième cas, la fréquence des échanges entre $O$ et $M$ est de 5 fois par seconde. La figure 19 est valable pour $U_{p}=-30 \%$ et $Z_{1}=0$, la figure 20 pour $U_{p}=-30 \%$ et $Z_{1}=-0,04(2 \mathrm{~Hz})$.

En bref, ces trois cas représentent les aspects essentiels de toutes les configurations possibles. On a déjà vu que le modèle original $(O)$ et le modèle modi-

\begin{tabular}{|c|c|c|c|c|c|}
\hline \multicolumn{5}{|c|}{ tableau III (pour les figures 17 et 18) } \\
\hline$t(\mathrm{sec})$ & $t \leqslant 1$ & $1<t \leqslant 3$ & $3<t \leqslant 6$ & $6<t \leqslant 10$ & $t>10$ \\
\hline $\begin{array}{c}\text { Modèle } \\
\text { du } \\
\text { procédé }\end{array}$ & $O$ & $M$ & 0 & $M$ & $O$ \\
\hline
\end{tabular}

*Remarque: Dans les tableaux II, III et dans les figures $15 \sim 20, O$ signifie le modèle Original du procédé qui correspond aux expressions (7.4) (7.6) tand is que $M$ signifie le modèle Modifié du procédé qui correspond aux expressions $(7.7) \sim(7.9)$. 


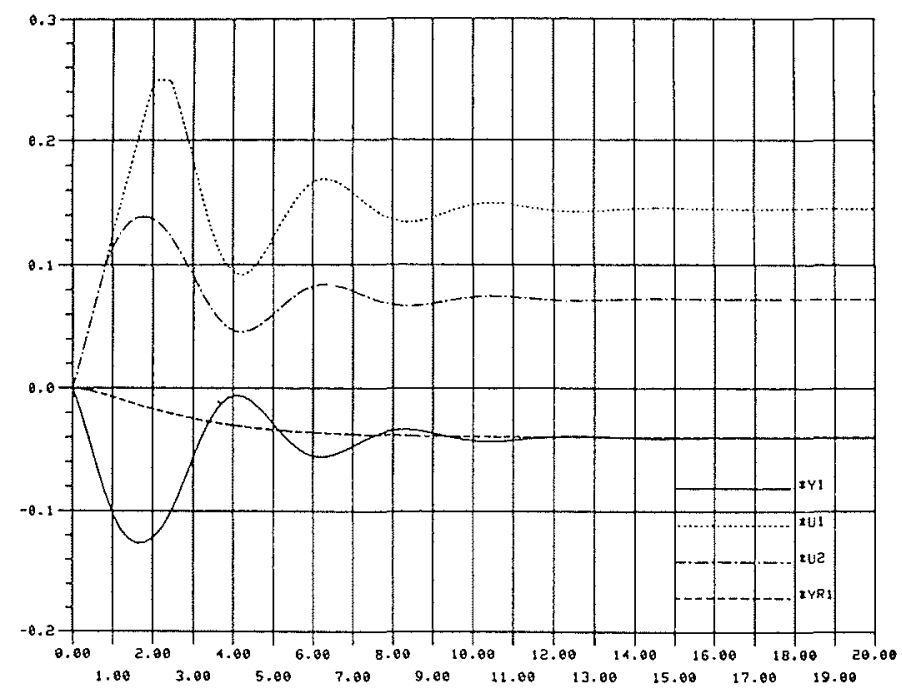

Figure 13 - Réponse du procédé modifié dans le cas où : $U P=30 \%$ et $Z 1=-0.04$.

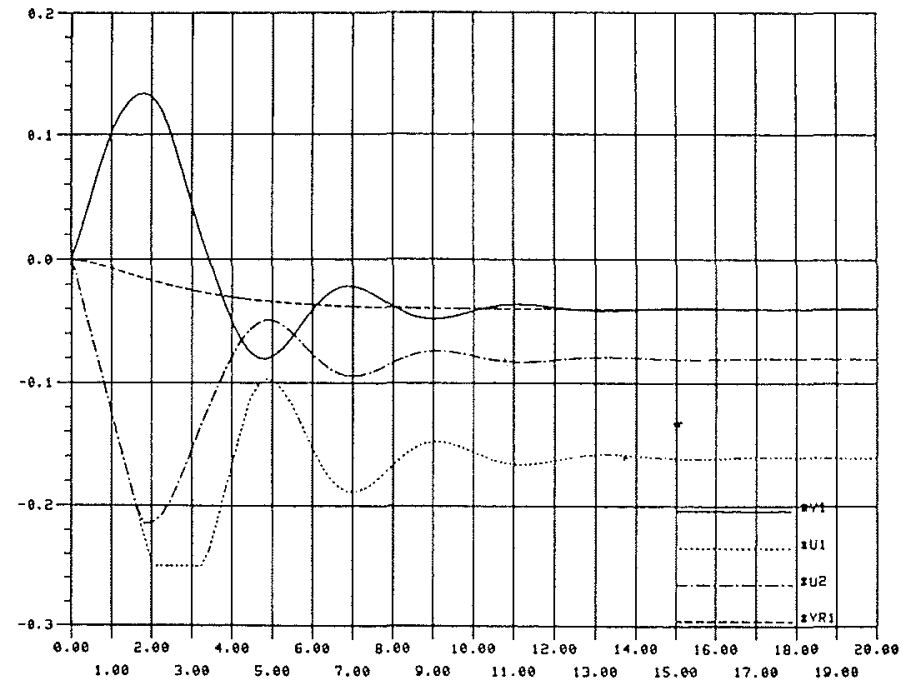

Figure 14 - Réponse du procédé modifié dans le cas où $U P=-30 \%$ et $Z 1=-0.04$

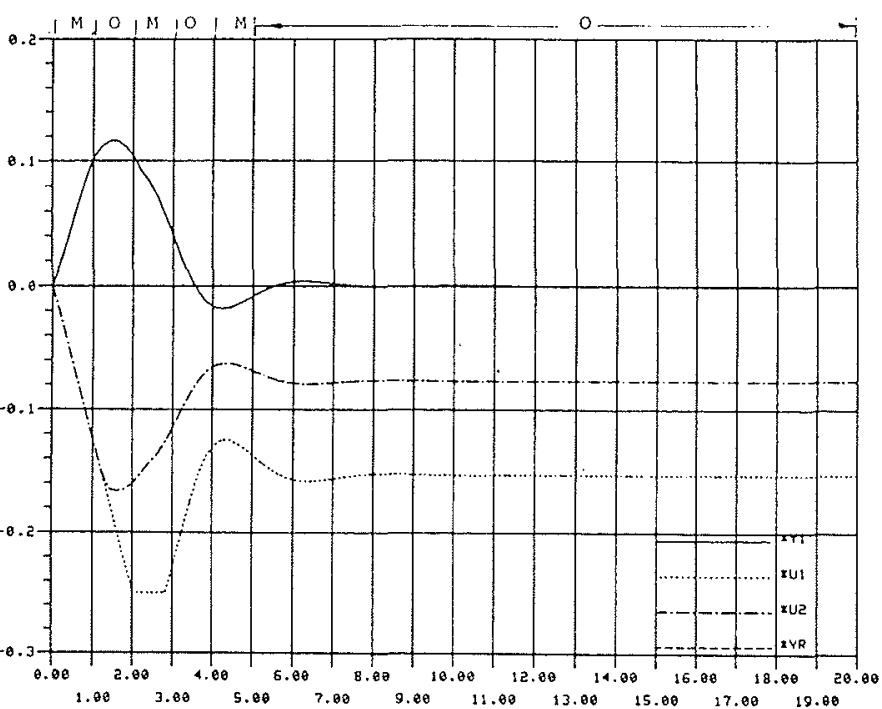

Figure 15 - Vérification de l'adaptibilité pour $U P=-30 \%$ et $Z 1=0$ (au tableau II).

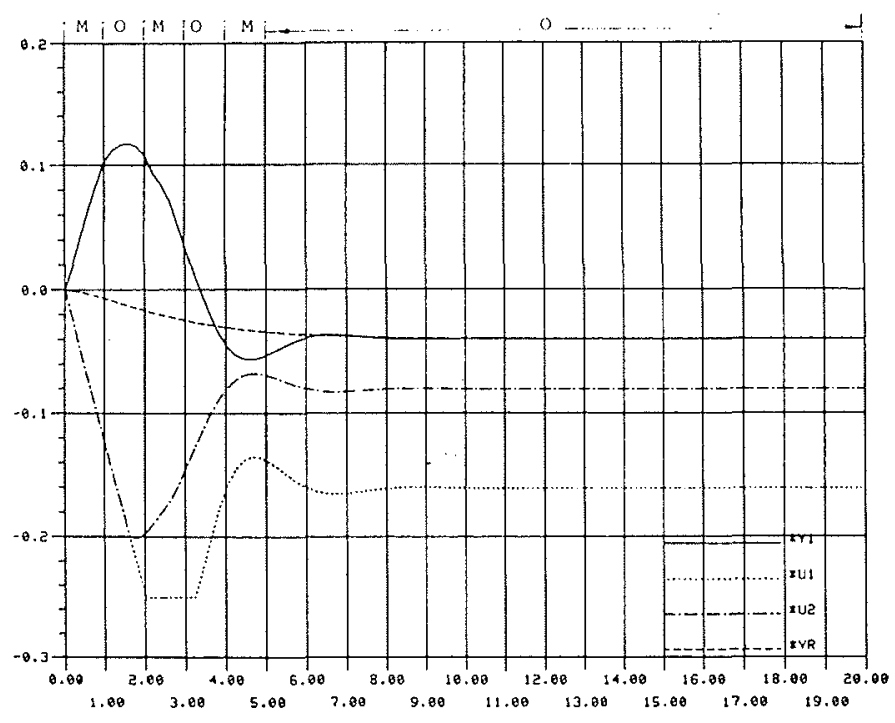

Figure 16 - Vérification de l'adaptibilité pour $U P=-30 \%$ et $Z 1=-.04$ (au tableau II).

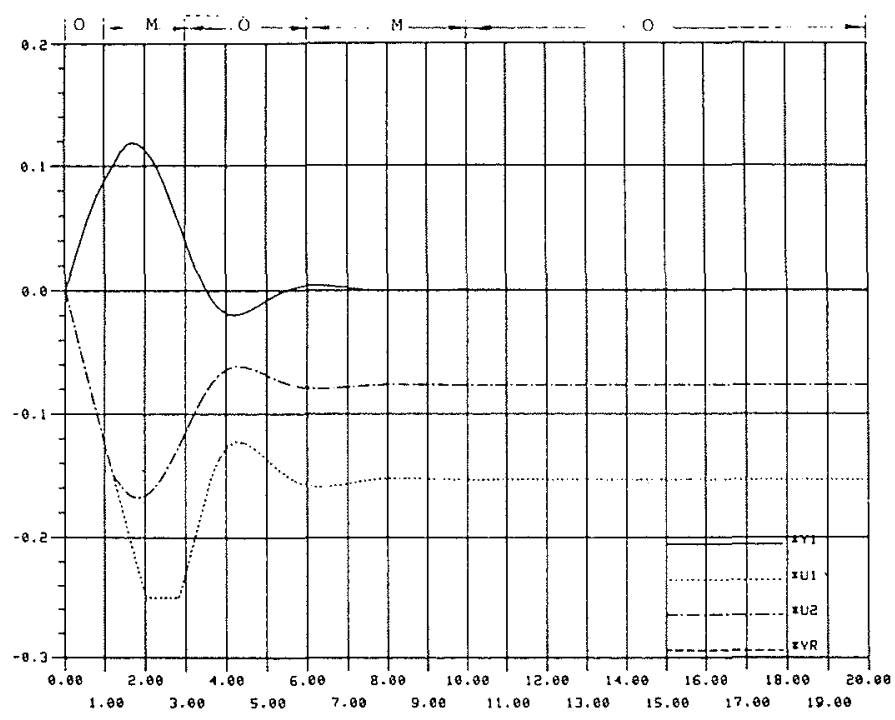

Figure 17 - Vérification de l'adaptibilité pour $U P=-30 \%$ et $Z 1=0$ (au tableau III).

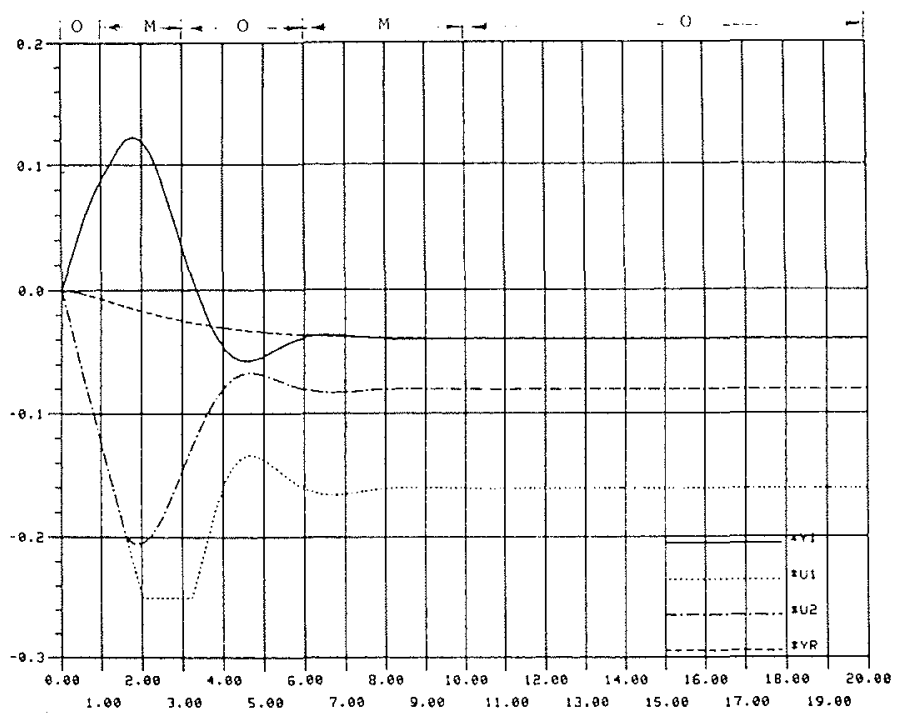

Figure 18 - Vérification de l'adaptibilité pour $U P=-30 \%$ et $Z 1=-.04$ (au tableau III). 


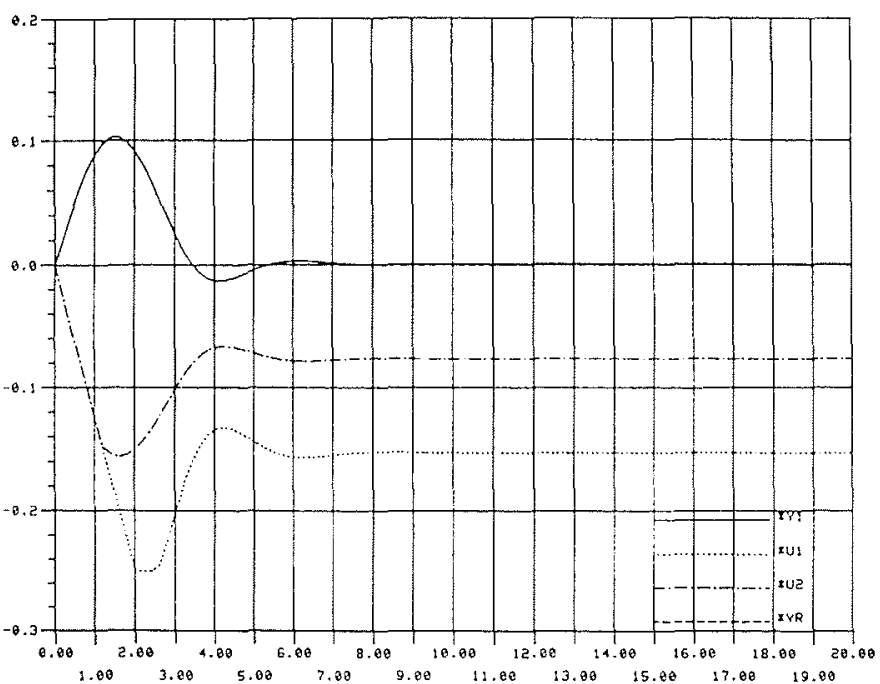

Figure 19 - Vérification de l'adaptibilité pour $U P=-0.3$ et $Z 1=0$, fréquence $O-M: 5 / \mathrm{sec}$.

fié $(M)$ sont très différents. Mais on se rappelle que pour les trois cas, la structure du système de commande et ses paramètres initiaux ne changent pas. On voit bien, sur les figures $15 \sim 20$ que les performances dans les trois cas sont satisfaisantes et qu'elles sont très proches de celles du modèle original.

Donc, il n'est pas difficile d'affirmer que le système étudié possède une bonne adaptabilité à la modification des paramètres du système à commander.

\section{Conclusion}

Par rapport au régulateur classique et à d'autres branches de la commande moderne, la méthode étudiée sur la commande adaptative multivariable numérique possède les avantages suivants :

1) Les paramètres du régulateur varient avec les régimes en assurant des performances les plus proches possibles des performances désirées déterminées par le modèle de référence. C'est le principal avantage de la commande adaptative par rapport au régulateur classique. Les simulations montrent que la méthode étudiée est d'une robustesse, d'une adaptabilité et d'une souplesse satisfaisantes.

2) La méthode étudiée assure à la fois l'optimalité dynamique et l'optimalité statique. Au point de vue

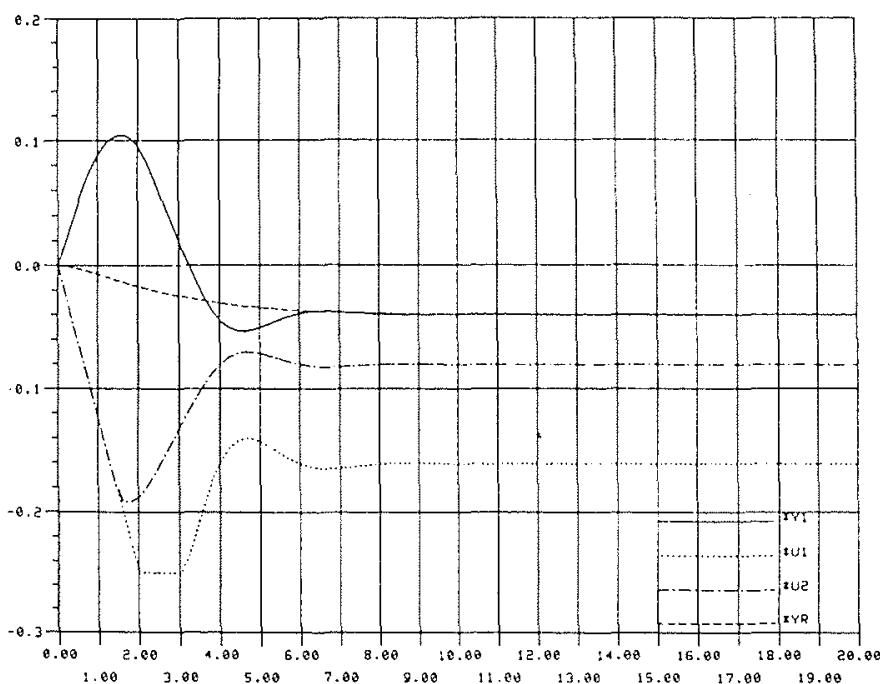

Figure 20 - Vérification de l'adaptibilité pour $U P=-.3$ et $Z 1=-.04$, fréquence $O \cdot M: 5 / \mathrm{sec}$.

dynamique, l'écart de fréquence est minimale ; au point de vue statique, ou plutôt énergétique, la conjugaison des deux organes de réglage est réalisée.

3) La prise en compte de la perturbation mesurable (prévision de la charge) améliore la performance.

4) La structure étudiée est implantable sur microprocesseur en ce qui concerne le volume de mémoire et le temps de calcul.

Cette réalisation par microprocesseur fera l'objet d'un autre document.

\section{Références}

[1] LANDAU I.D. - Sur une méthode des sytèmes adaptatifs avec modèles utilisés pour la commande et l'identification d'une classe de procédés physiques. Thèse d'Etat, Grenoble, 1973.

[2] ASTRÖM K.J. and WITTENMARK B. - On self Tuning Regulator - Automatica, Vol. 9, pp. 185-199, 1973.

[3] IRVING E. and DANG VAN MIEN - Discrete-time Model References Multivariable Adaptative Control. Application to Electrical Power Plants.

4 Conférence internationale sur l'analyse et l'optimisation des systèmes, 1980.

[4] YE LUQING et BORNARD G. - Régulation et commande de la fréquence du groupe hydro-électrique. Note interne LAG. Grenoble, 1980 . 